\title{
Peutz-Jeghers syndrome: an unusual cause of recurrent intussusception in a 7-year-old boy
}

\author{
Sinan Kılıç ${ }^{1}$, Ahmet Atıc1 ${ }^{1}$, Özlem Soyköse-Açıkalın² \\ Departments of ${ }^{1}$ Pediatric Surgery, and ${ }^{2}$ Pathology, Aksaray Government Hospital, Aksaray, Turkey. \\ E-mail: dr.sinankilic@yahoo.com \\ Received: 17th February 2016, Revised: 5th May 2016, Accepted: 17th May 2016
}

SUMMARY: Kılıç S, Atıcı A, Soyköse-Açıkalın Ö. Peutz-Jeghers syndrome: An unusual cause of recurrent intussusception in a 7-year-old boy. Turk J Pediatr 2016; 58: 535-537.

Peutz-Jeghers syndrome (PJS) is an autosomal dominant inherited disorder characterized by intestinal hamartomatous polyps in association with mucocutaneous pigmentations. Main symptoms of PJS in childhood are abdominal pain, obstruction, intussusception, and bleeding from hamartomatous polyps. PJS carries a high risk of gastrointestinal cancer with advancing years. Although, intussusception has been reported as a well-known complication of PJS, recurrent intussusception as an alarming finding in a patient with normal gastrointestinal endoscopy is uncommon. A 7-year-old boy who had recurrent intussusception episodes and diagnosed with PJS histopathological confirmation after surgical excision of involved bowel segments is presented to discuss the clinical features and treatment options of recurrent intussusception as a presenting finding of PJS.

Key words: Peutz-Jeghers syndrome, intussusception, cancer, child.

Peutz-Jeghers syndrome (PJS) is an inherited disorder characterized by hamartomatous polyps in the gastrointestinal tract and pigmented mucocutaneous lesions. It was first reported by Peutz in $1921^{1}$. Syndrome's clinical description was written by Jeghers in $1949^{2}$. In 1954, Bruwer "coined" the eponym "Peutz-Jeghers" PJS is a well-known but rare disease. The incidence is 1 in 30,000 to 120,000 live births and both sexes affected equally ${ }^{4}$. Most patients have a characteristic clinical course of recurrent episodes of polyp induced bowel obstruction and bleeding. In addition to the clinical diagnosis and polyp-related complications such as intestinal obstruction (14\%), abdominal pain $(23 \%)$, rectal bleeding (14\%), and polyp intussusception (40\%), the most challenging clinical aspect is early tumor occurrence or preinvasive lesion identification ${ }^{5,6}$. Also, risk of gastrointestinal and extra-gastrointestinal malignancies is significantly increased in PJS patients ${ }^{7,8}$.

Typical clinical features such as abdominal pain, hyperpigmentous lip spots and polypoid lesions in gastrointestinal endoscopy are commonly present in most of the patients. Although, these clinical findings suggest PJS, identification of hamartamatous polys in endoscopy and histopathologic confirmation is mandatory for diagnosis.

\section{Case Report}

A 7-year-old boy was admitted to emergency with complaints of abdominal pain and vomiting lasting three days. He had a history of recurrent colicky abdominal pain for approximately six months. There was no history of hematemesis or melena, and significant family history. He had underwent upper gastrointestinal endoscopy and colonoscopy because of multiple hyperpigmented macules over only the lips (Fig. 1), 4 months prior from this admission which revealed normal endoscopic findings.

On admission, his vital findings were within normal limits and physical examination showed slight distension with generalized tenderness. Plain abdominal radiography was remarkable with small air/fluid levels. Blood count analysis indicated leukocytosis (white blood cell 15,300/ $\mathrm{mm}^{3}$ ), and hiponatremia (Na: $\left.132 \mathrm{mEq} / \mathrm{L}\right)$. 


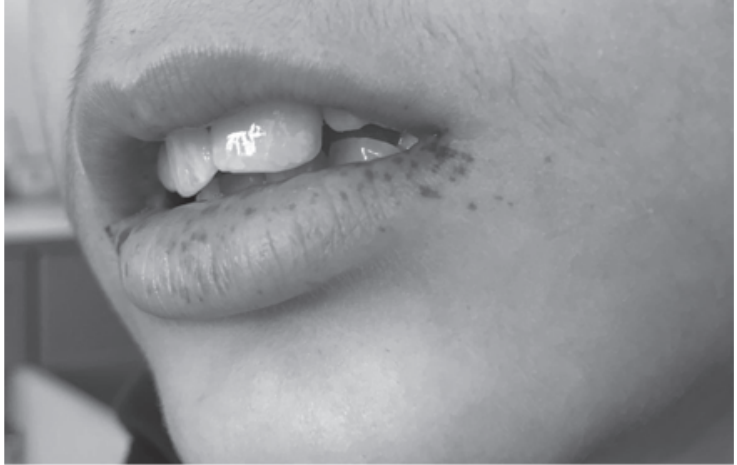

Fig 1. Mucocutaneus pigmentation over and around the lip.

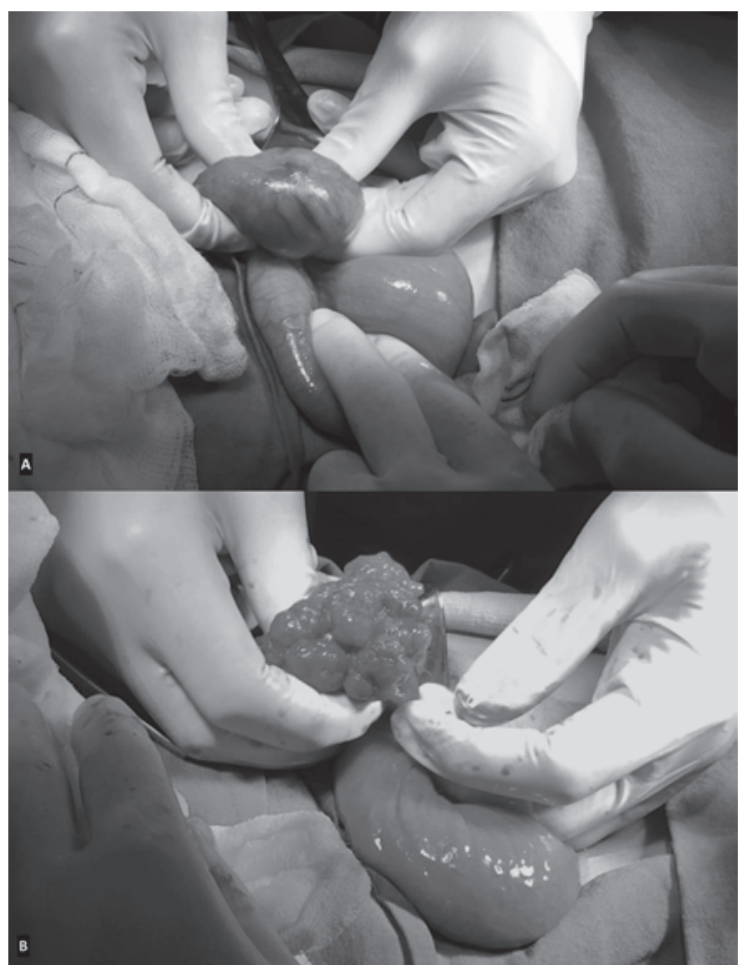

Fig 2. (a) Appearance of jejuno-jejunal intussusception after the exploration. (b) Hamartomatous polyp in the small bowel.

Urine analysis and the other blood biochemistry were normal. Abdominal ultrasonography revealed the typical "target sign" and the patient was diagnosed as small bowel intussusception at left upper quadrant of the abdomen. According to physical examination findings and bowel obstruction, he underwent explorative laparotomy. A jejuno-jejunal intussusception was found $5 \mathrm{~cm}$ distal of the ligament of Treitz during surgical exploration (Fig. 2A). Intussusception could not reduced manually and a polypoid mass in the jejunal lumen was

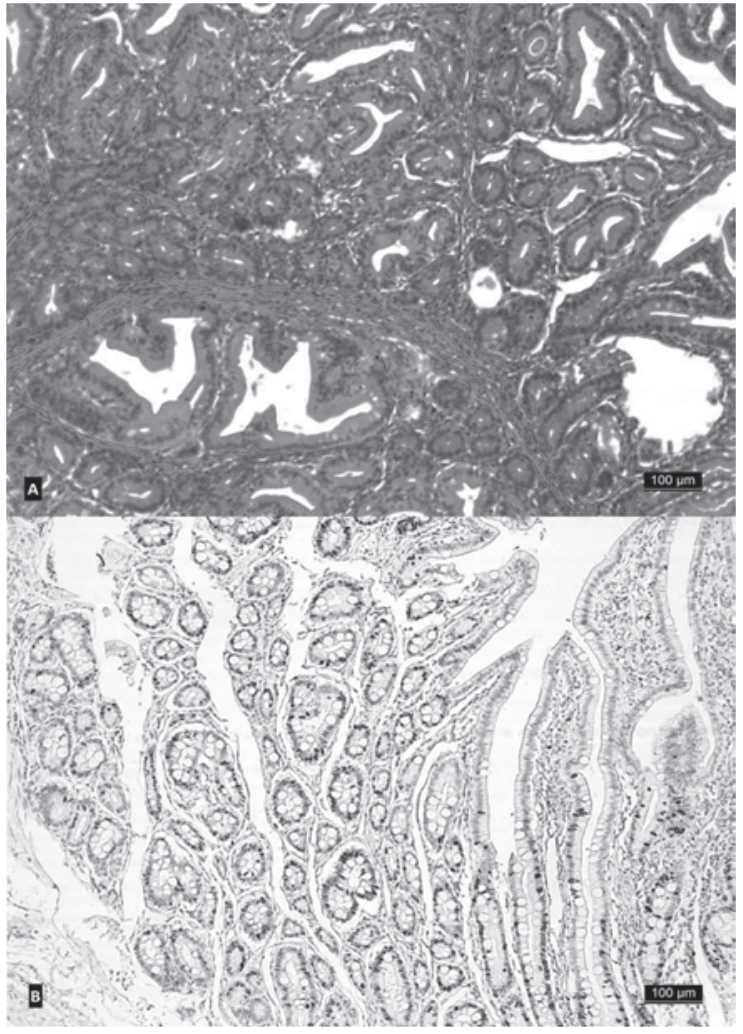

Fig 3. (a) Sparse muscularis mucosae covered with normal villi demonstrated (HE, X40). (b) Cells with atypical nucleus indicates low-grade dysplasia which were seen in stroma (Ki-67, X20).

the leading point (Fig. 2B). During the surgical examination, there weren't any other polyps at the rest of bowel. The intussuscepting segment including the polyp was excised by small bowel resection, and end-to-end anastomosis was performed.

Macroscopically, the polyp was approximately $5.5 \times 5 \times 4 \mathrm{~cm}$ in size and histopathological examination of the specimen revealed hamartamatous polyp. The polyp encountered in the small bowel had sparse muscularis mucosae covered with normal villi and was defined as a hamartoma (Fig. 3A). PeutzJeghers syndrome was confirmed histologically with low-grade dysplasia (Fig. 3B). After the operation the boy was discharged from the hospital uneventfully. He is still being followed up closely. There was no family history for the PJS. Consequently the patient and his family were referred to a tertiary medical center for further genetic and endoscopic investigations.

Written informed consent was obtained from patients family. 


\section{Discussion}

Peutz-Jeghers syndrome is a hereditary disease characterized with gastrointestinal polyposis and skin or mucosal pigmentation. Polyps found most frequently in the small bowel; besides can occur in the stomach, colon and rectum. Polypoid lesions are almost hamartomatous and are not usually precancerous. The definition requires histopathological confirmation of hamartomatous gastrointestinal polyps and two of the following features: small bowel polyposis, positive family history and pigmented skin or mucosal brown macules. Our patient underwent upper and lower endoscopy with presumptive diagnosis of PJS before the clinical findings of intussusception. Endoscopic evaluation revealed normal findings without any polypoid lesion. Despite normal endoscopic findings, intussusception developed by a polyp and diagnosis was confirmed by the help of histopathologic evaluation of the excised bowel. The boy has no family history of PJS or intestinal polyps. It has been reported that approximately $50 \%$ of cases are sporadic and represent new mutations.

Although the association of PJS and intussusception is well-known, this complication is rarely a presented in finding of PJS. Polyps are a common cause of intussusception and lead to small bowel obstructions. Recurrent intussusception requires further evaluations to define leading points. Patients with hyperpigmentous lip lesions with recurrent intussusception episodes may require surgical explorations not only relieving small bowel obstruction but also to diagnose the underlying polypoid lesions.

Most patients having recurrent episodes of polyp induced bowel intussusception and require clinical observation. Patients with PJS should be regularly and closely monitored in order to reduce the number of laparotomies and due to the increased risk of cancer. Since these patients have an increased risk of malignant disease in the gastrointestinal system, further evaluations are needed during close follow-up. Upper and lower endoscopy is recommended every two years from the age of 10 in the is patient group ${ }^{9}$. Recent advances in genetic testing and capsule endoscopy should result in improved management of patients with PJS. In addition, these patients should be included in specific follow-up programs with screening for pancreatic cancer in the future ${ }^{10}$.

In conclusion, recurrent intussusception may be a finding suggests ruling out PJS even in patients with normal gastrointestinal endoscopic evaluations. Patients with other signs of PJS, it may require early surgical exploration in case of intussusception. It should be kept in mind that histopathological confirmation of PJS can be obtained only after surgical excision.

\section{REFERENCES}

1. Peutz JLA. Very remarkable case of familial polyposis of mucous membrane of intestinal tract and accompanied by peculiar pigmentations of skin and mucous membrane. Nederl Maandschr Geneesk 1921; 10: 134-146.

2. Jeghers H, McKusick VA, Katz KH. Generalized intestinal polyposis and melanin spots of the oral mucosa, lips and digits. A syndrome of diagnostic significance. N Engl J Med 1949; 241: 993-1005.

3. Bruwer A, Bargen JA, Kierland RR. Surface pigmentation and generalized intestinal polyposis (Peutz-Jeghers syndrome). Proc Staff Meet Mayo Clin 1954; 29: 168171.

4. Lindor NM, Greene MH. The concise handbook of family cancer syndromes. Mayo Familial Cancer Program. J Natl Cancer Inst 1998; 90: 1039-1071.

5. Mehenni H, Resta N, Guanti G, et al. Molecular and clinical characteristics in 46 families affected with Peutz-Jeghers syndrome. Dig Dis Sci 2007; 52: 19241933.

6. Schreibman IR, Baker M, Amos C, McGarrity TJ The hamartomatous polyposis syndromes: a clinical and molecular review. Am J Gastroenterol 2005; 100: 476-490.

7. Boardman LA, Thibodeau SN, Schaid DJ, et al. Increased risk for cancer in patients with the Peutz-Jeghers syndrome. Ann Intern Med 1998; 128: 896-899.

8. Hemminki, A. The molecular basis and clinical aspects of Peutz-Jeghers syndrome. Cell Mol Life Sci 1999; 55: 735-750.

9. Giardiello FM, Trimbath JD. Peutz-Jeghers syndrome and management recommendations. Clin Gastroenterol Hepatol. 2006; 4: 408-415.

10. Latchford A, Greenhalf W, Vitone LJ, Neoptolemos JP Lancaster GA, Phillips RK. Peutz-Jeghers syndrome and screening for pancreatic cancer. Br J Surg 2006; 93: $1446-1455$. 